\title{
Synthesis of 3,3'-[methylenebis(3,1-phenylenenitrilo)]bis[1,3-dihydro]-2H-indol-2-one as a novel bis-Schiff base
}

\section{A. A. Jarrahpour*, D. Khalili}

Department of Chemistry, College of Science, Shiraz University, Shiraz 71454, Iran

Tel. +98 711 2284822, Fax: +98 711 2280926. e-mail: jarrah@chem.susc.ac.ir and aliasghar6683@yahoo.com

Received: 2 June 2005 / Accepted: 22 September 2005 / Published: 1 October 2005

Keywords: isatin, bis- Schiff base, 3,3'-diaminodiphenylmethane,

Isatin (1H-indole-2,3-dione) was first obtained by Erdman and Laurent in 1841 as a product from the oxidation of indigo by nitric and chromic acids [1]. The synthetic versatility of isatin has led to the extensive use of this compound in organic synthesis. In nature, isatin is found in plants of the genus Isatis [2], in Calanthe discolor LINDL [3]. Isatin is the biologically active chemical produced by an Altermones sp. Strain inhabiting the surface of embryos of the cardiean shrimp Palaemon macrodectylus, which protect them from the pathogenic fungus Lagenidium callinectes [4]. Schiff bases of isatin were reported to possess anti-HIV [5-7], anticonvulsant [8], antibacterial [9-11], antiprotozoal [12,13], antifungal [14-16], antiviral [17-19] and antihelminthic [20,21] activities. The Schiff bases of isatin have also been used as a ligand for complexation of metals such as copper (II) [22]. Based on these facts, we decided to synthesize a new isatin bis-Schiff base as potential biological and complexometric agents. Its biological activities and analytical works are under study.<smiles>O=C1Nc2ccccc2C1=O</smiles>

1<smiles>Nc1cccc(Cc2cccc(N)c2)c1</smiles>

2
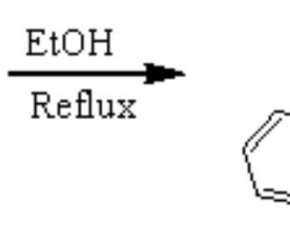

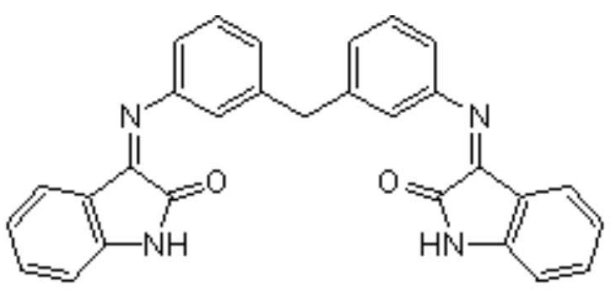

3

Isatin 1 (2.00 g, $13.6 \mathrm{mmol})$ and 3,3'-diaminodiphenylmethane 2 (1.35 g, $6.8 \mathrm{mmol})$ were dissolved in 35 $\mathrm{mL}$ of warm ethanol containing $0.45 \mathrm{~mL}$ of acetic acid. The reaction mixture was refluxed for $17 \mathrm{~h}$ and set aside. The resultant solid was filtered and washed with ethanol. Pure Schiff base $\mathbf{3}$ was obtained upon recrystallization from ethanol $(2.40 \mathrm{~g}, 77.3 \%)$.

Melting point: $>260^{\circ} \mathrm{C}$

IR $\left(\mathrm{KBr}, \mathrm{cm}^{-1}\right): 1652(\mathrm{C}=\mathrm{N}) ; 1726.2(\mathrm{C}=\mathrm{O}) ; 3168.8(\mathrm{~N}-\mathrm{H})$.

${ }^{1} \mathrm{H}-\mathrm{NMR}\left(250 \mathrm{MHz}, \mathrm{DMSO}-d_{6}\right): \delta=10.96(1 \mathrm{H}, \mathrm{s}, \mathrm{N}-\mathrm{H}) ; 7.43-6.29(16 \mathrm{H}, \mathrm{m}, \mathrm{ArH}) ; 4.07\left(2 \mathrm{H}, \mathrm{s}, \mathrm{CH}_{2}\right)$.

${ }^{13} \mathrm{C}-\mathrm{NMR}\left(62.9 \mathrm{MHz}, \mathrm{DMSO}-d_{6}\right): \delta=72.54 ; 115.63 ; 116.39 ; 119.11 ; 120.04 ; 121.65 ; 122.25 ; 124.28$; $126.41 ; 127.20 ; 130.18 ; 130.35 ; 134.66 ; 139.19 ; 147.51 ; 151.73 ; 155.53 ; 159.76 ; 168.29$.

MS (m/z, \%): 457 (17.40); 456 (34.40); 327 (12.40); 312 (31.40); 299 (14.20); 284 (23.40); 44 (100.00). 


\section{Acknowledgment}

The authors are grateful to Shiraz University Research Council for financial support (Grant No.83GR-SC-31 and 84-GR-SC-23 ).

\section{References}

1. Dasilva, J. F. M.; Garden, S. J.; Pinto, A. C. J. Braz. Chem. Soc. 2001, 12(3), 273.

2. Guo, Y.; Chen, F.; Zhongcaoyao 1986, 17, 8. (CA 104:213068f)

3. Yoshikawa, M.; Murakami, T.; Kishi, A.; Sakurama, T.; Matsuda, H.; Nomura, M.; Matsuda, H.; Kubo, M. Chem. Pharm. Bull. 1998, 46, 886.

4. Gil-Turners, M. S.; Hay, M. E.; Fenical, W. Science, 1989, 116.

5. Pandeya, S. N.; Yogeeswari, P.; Sriram, D.; De Clercq, E.; Pannecouque, C.; Witvrouw, M. Chemotherapy, 1999, 45, 192-196.

6. Pandeya, S. N.; Sriram, D.; Nath, G.; De Clercq, E. Eur. J. Med. Chem. 2000, 35, 249-255.

7. Pandeya, S. N, Sriram, D.; Nath, G.; De Clercq, E. Arzneimittel Forschun./Drug Res. 2000, 50, 55-59.

8. Sridhar, S. K.; Pandeya, S. N.; Stables, J. P.; Armes, S. K. Eur. J. Pharm. Sci. 2002, 16, 129.

9. Pandeya, S. N.; Sriram, D. Acta Pharm. Turc. 1998, 40, 33.

10. Sarangapani, M.; Reddy, V. M. Indian J. Pharm. Sci. 1994, 56, 174.

11. Varma, R. S.; Nobles, W. L. J. Pharm. Sci. 1975, 64, 881.

12. Imam, S. A.; Varma, R. S. Experientia, 1975, 31, 1287.

13. Varma, R. S.; Khan, I. A. Polish J. Pharmacol. Pharm. 1977, 29, 549.

14. Pandeya, S. N.; Sriram, D.; Nath, G.; De Clercq, E. Indian J. Pharm. Sci. 1999, 61, 358.

15. Pandeya, S. N.; Sriram, D.; Nath, G.; De Clercq, E. Sci.Pharm. 1999, 67, 103.

16. Pandeya, S. N.; Sriram, D.; Nath, G.; De Clercq, E. Pharm. Acta Helv. 1999, 74, 11.

17. Varma, R. S.; Nobles, W. L. J. Med. Chem. 1967, 10, 972.

18. Singh, S. P.; Shukla, S. K.; Awasthi, L. P. Curr. Sci. 1983, 52, 766.

19. Logan, J. C.; Fox, M. P.; Morgan, J. M.; Makohon, A. M.; Pfau, C. J. J. Gen. Virol. 1975, $28,271$.

20. Sarciron, S. E.; Audin, P.; Delebre, I.; Gabrion, C.; Petavy, A. F.; Paris, J. J. Pharm. Sci. 1993, 82, 605.

21. Et-savi, E. A.; Mostafa, T. B.; Mostafa, B. B. J. Egypt. Soc. Parasitol. 1998, 28, 481.

22. Cerchiaro, G.; Micke, G. A.; Tavares, M. F. M.; Ferreira, A. M. D. C. J. Mol. Catal. A: Chem, 2004, $221,29$.

Sample Availability: Available from MDPI.

(C) 2005 MDPI. All rights reserved. 